\title{
Thermomechanical Modeling and Analysis of Workpiece in End Milling Process
}

\author{
Yong Feng ${ }^{1, a^{*}}$, Binghui Jia ${ }^{1, b}$, Guodong Yan $^{1, c}$ and Xiaolin Jia ${ }^{1, d}$ \\ ${ }^{1}$ School of Mechanical Engineering, Nanjing Institute of Tech., Nanjing 211167, China. \\ afengyong007@sina.com, ${ }^{\mathrm{b}}$ jiabinghuixyz@163.com, ${ }^{\mathrm{c}}$ ygd0537@126.com, ${ }^{\mathrm{d}}$ 1535347917@qq.com
}

Keywords: Oblique cutting, End milling, AISI1045 steel.

Abstract. In order to study the cutting force distribution of the workpiece during the process of vertical milling machining, the thermomechanical model of the steady oblique cutting is derived. The measuring system of cutting force is also established, and then the feasibility of theoretical model is verified. First, according to the steady oblique cutting of cutting force and cutting temperature characteristics, coupled thermomechanical model of the workpiece in the milling process is derived. Based on above, a dynamic solving approach for cutting force is proposed. Then, the dynamic measurement system of force is established by using artificial Kistler force measurement. Finally, the thermomechanical model and dynamic measurement system are used to analyze the cutting force of AISI1045 steel under specific conditions. Analysis results indicate that the maximum of $F x, F y$ and $F z$ are $198 \mathrm{~N}, 40 \mathrm{~N}$ and $90 \mathrm{~N}$ respectively, the error between calculated and measured value are $2 \%, 20 \%$ and $10 \%$ respectively. It shows that the established method can be used to investigate the thermo-mechanics of workpiece accurately in milling process.

\section{Introduction}

Because existing complicated thermal, dynamical and couple effects, it is hard to establish accurate thermal-dynamic model. Hence, it should make some reasonable simplification to establish its thermal-dynamic model in milling process ${ }^{[1]}$. Then, fairly accurate analysis of the change of the real-time physical quantities such as temperature, stress and strain can be done.

Thermal-dynamic modeling of oblique cutting is widely studied by scholars all over the world. Thermal-dynamic model of orthogonal cutting process is put forward by OXLEY ${ }^{[2]}$, and rakecasting analysis is done under the assumption that cutting force is ignorant of oblique cutting angle and flow cutting angle. MOUFKI et.al ${ }^{[3]}$ took fully account of thermal-dynamic couple effect which would affect cutting formation, and put forward calculation method of flow cutting angle. $\mathrm{WU}^{[4]}$ gave theoretical formation of oblique cutting force by coordination transfer method. Research above was mainly on milling force and temperature of oblique milling respectively, but are not on relationship of each other. Present study can't build effective relation between milling force and temperature, thus can't reflect dynamic process of milling force versus rotation angle. Based on oblique milling force model, 3-D milling force calculation model of end milling process is built by micro element discretion in this paper. Moreover, the relation between milling force rakecast model and temperature rakecast model is build. Based on above, periodical varied condition of cutting force during milling process are analyzed, which provide accurate basis for high speed milling work analysis and optimization.

\section{Cutting Force Model}

Geometric Relationship. The Fig.1 2 show the geometric model of oblique cutting process ${ }^{[5]}$. Where, $x_{\mathrm{n}}$ axis of plane $P_{\mathrm{n}}$ is vertical to the cutting edge and locates inside the cutting plane $P_{\mathrm{S}}$. $z_{\mathrm{n}}$ axis is coincide with cutting edge, and $y_{\mathrm{n}}$ axis is vertical to plane $P_{\mathrm{n}} . x_{0}$ axis of cutting plane $P_{\mathrm{S}}$ is opposite to cutting speed. $z_{0}$ axis is parallel to the radial cutting width $w . y_{0}$ axis is vertical to the cutting plane $P_{\mathrm{S}}, y_{0}$ axis and $z_{0}$ axis formed the reference plane $P_{0}$. Other related cutting plane has shear plane $P_{\text {sh }}$, rakeface $A_{\gamma}$ and equivalent plane $P_{\mathrm{e}}$. The equivalent plane $P_{\mathrm{e}}$ is determined by the cutting speed $v_{\mathrm{c}}$ 
and chip speed $v_{\mathrm{e}}$. The cutting mechanism of $P_{\mathrm{e}}$ can be regarded as the accumulation of 2-D cutting state.

In addition, angles about oblique cutting haveinclination angle $\lambda_{\mathrm{s}}$, rake angle $\gamma_{\mathrm{n}}$ of planar $P_{\mathrm{n}}$ of c utting tool, the shear angle $\Phi_{\mathrm{n}}$ in normalplane,chip flow angle $\eta_{\mathrm{c}}$ of rake face $A_{\gamma}$ and the shear flowan gle $\eta_{\mathrm{s}}$ in shear plane $P_{\mathrm{sh}}$.

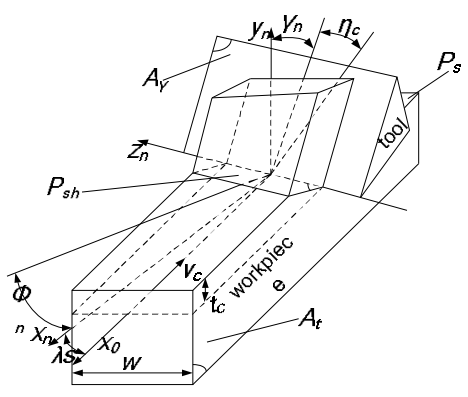

Fig. 1: Oblique Cutting Process

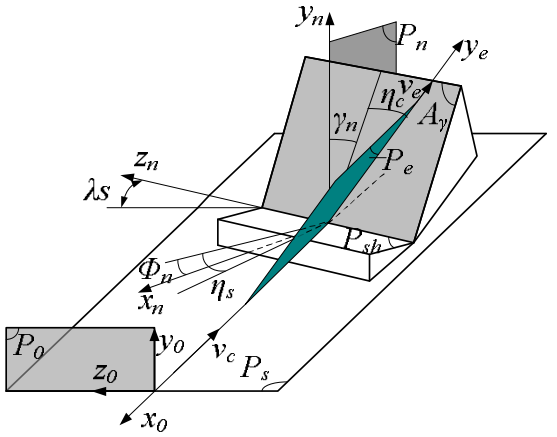

Fig. 2: Geometric Relationships

Equivalent plane orientation is determine by the equivalent plane Angle $\eta_{\mathrm{e}}$.

$\tan \eta_{e}=\frac{\tan \eta_{c} \cos \eta_{s}+\sin \left(\phi_{n}-\gamma_{n}\right) \sin \eta_{s}}{\cos \left(\phi_{n}-\gamma_{n}\right)}$

According to the geometrical relationship, the flow Angle of $\eta_{\mathrm{s}}$ can be express as below $\tan \eta_{s}=\frac{\tan \lambda_{s} \cos \left(\phi_{n}-\gamma_{n}\right)-\tan \eta_{c} \sin \phi_{n}}{\cos \gamma_{n}}$

Where, $\eta_{c}$ — chip flow Angle (rad), using Stabler's test rules, $\eta_{c}=\lambda_{s}$.

$\Phi_{n} \longrightarrow$ normal shear Angle (rad), applying model given by Armarego, $\Phi_{n}=\pi / 4-\left(\beta_{n}-\gamma_{n}\right)$.

$\beta_{n} \longrightarrow$ normal friction Angle (rad).

$r_{c}$ is a ratio between the cutting thickness of $t_{\mathrm{c}}$ and chip thickness $t_{\mathrm{ch}}$, obtained generally by the test data.

$r_{c}=\frac{\sin \phi_{n}}{\cos \left(\phi_{n}-\gamma_{n}\right)}=\frac{t_{c h}}{t_{c}}$

Velocity Relationship. Shear line vectorp in equivalent plane can be got by unit normal vector $\mathrm{m}$ of the shear plane $P_{s h}$ and the unit normal vector $n$ of equivalent planar $P_{e}$. According to the equation(4), it can be obtained. Where, the unit quantity $m$ can be got by clock wisely revolving around the z axis $\Phi_{n}$. A unit vector $n$ can be got by clock wisely revolving around the $x$ axis $\eta_{c}$. So the relation of the shear Angle $\Phi_{e}$ ofthe equivalent plane can be obtained assin $\Phi_{e}=\sin \Phi_{n} \cos \eta_{\mathrm{c}}$; The relationship about shear length $L_{e}$ of the equivalent plane is $L_{e}=t_{c} / \sin \Phi_{n} \cos \eta_{\mathrm{c}}$.

$p=m \times n=\left(\begin{array}{c}\cos \phi_{n} \cos \eta_{c} \\ \sin \phi_{n} \cos \eta_{c} \\ \sin \phi_{n} \sin \eta_{c}\end{array}\right)$

Chip velocity component in upward thickness direction of the shear zone is constant value ${ }^{[6]}$. After taking into the boundary conditions, the shear velocity $v_{\mathrm{s}}$, chip velocity $v_{\mathrm{e}}$, and shear plane area $A_{\mathrm{sh}}$ of equivalent plane can be express as equation (5), (6) and (7).

$v_{e}=v_{c} \frac{\cos \lambda_{s} \sin \phi_{n}}{\cos \eta_{c} \cos \left(\phi_{n}-\gamma_{n}\right)}$

$v_{s}=v_{c} \frac{\cos \lambda_{s} \cos \gamma_{n}}{\cos \eta_{s} \cos \left(\phi_{n}-\gamma_{n}\right)}$

$A_{s h}=\frac{w t_{c}}{\cos \lambda_{s} \sin \phi_{n}}$

Force Relationship. According to the relation of cutting force in oblique cutting process, the mechanics model of oblique cuttingis more complex than the model of orthogonal cutting, but the 
mechanics model of cutting plane is wholly same with the situation of orthogonal cutting. The relationship of cutting force in normal plane can be obtain as equation $8 \sim 10$.

$$
\begin{aligned}
& F_{t n}=\tau_{s} w t_{c} \frac{\cos \eta_{s} \cos \left(\beta_{n}-\gamma_{n}\right)}{\sin \phi_{n} \cos \lambda_{s} \cos \left(\phi_{n}+\beta_{n}-\gamma_{n}\right)} \\
& F_{a n}=\tau_{s} w t_{c} \frac{\cos \eta_{s} \sin \left(\beta_{n}-\gamma_{n}\right)}{\sin \phi_{n} \cos \lambda_{s} \cos \left(\phi_{n}+\beta_{n}-\gamma_{n}\right)} \\
& F_{r n}=F_{s} \sin \eta_{s}=\tau_{s} w t_{c} \frac{\sin \eta_{s}}{\sin \phi_{n} \cos \lambda_{s}}
\end{aligned}
$$

Considering the influence of inclination angle $\lambda \mathrm{s}$, the cutting force of main plane can be get after coordination transformation.

$$
\left[\begin{array}{l}
F_{x} \\
F_{y} \\
F_{z}
\end{array}\right]=\left[\begin{array}{ccc}
\cos \lambda_{s} & 0 & \sin \lambda_{s} \\
0 & 1 & 0 \\
-\sin \lambda_{s} & 0 & \cos \lambda_{s}
\end{array}\right]\left[\begin{array}{l}
F_{t n} \\
F_{a n} \\
F_{r n}
\end{array}\right]
$$

\section{Material Constitutive Model}

Cutting process is a thermal process with a high strain rate, high shear rate and the high rising temperature. Whether thethermal-mechanical characteristic equation of workpiece material is correct or not will directly affect the accuracy of cutting parameter prediction. In this article, the workpiece material as a viscous plastic solid isotropic material, its thermal properties can be describedby Johnson-Cook model. Introducing it into primary shear zone boundary conditions, simplified constitutive model can be obtained as shown as equation $12^{[8]}$.

$\tau=\rho\left(v_{c} \cos \lambda_{s} \sin \phi_{n}\right)^{2} \gamma+\tau_{0}$

Where, $\rho$ material density $\left(\mathrm{kg} \cdot \mathrm{m}^{-3}\right)$.

$\tau_{0} \_$shear stress when just coming into the shear zone $(\mathrm{Pa})$.

$\gamma-$ strain rate ${ }^{[9]}$, can be approximated as $\gamma=\frac{\cot \phi_{n}+\tan \left(\phi_{n}-\gamma_{n}\right)}{\cos \eta_{s}}$.

\section{Experimental Study}

Experimental Principle. Measuring principle of milling force and temperature is shown in Fig.3 ${ }^{[10-11]}$, the coordinate origin in the system is located in the position of lines in the top right the artifacts. $\mathrm{x}, \mathrm{z}$ axis is shown in the figure, $\mathrm{y}$ axis is determined by the right-hand rule. Kistler 9257B dynamometer is used to measure the three-dimensional cutting force in milling process. The dynamometer is connected with artifacts through the four bolts, and recycling splint is fixed on the machine tooling platform to ensure smooth dynamometer in the cutting process.

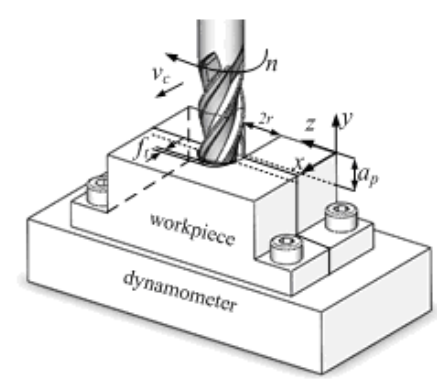

Fig. 3:Cutting Test Principle

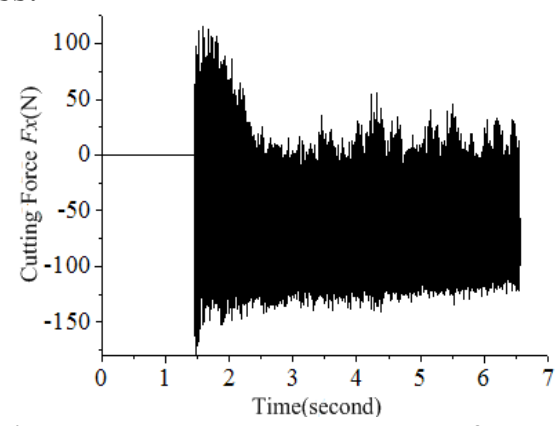

Fig.4: Measurement Curve of $F_{\mathrm{x}}$

Experimental System. Machine tool is vertical milling machining center MCV850, the highest spindle speed is $50 \sim 8000 \mathrm{rpm}$. Material of workpiece is AISI1045 steel; Specimen size is $80 \times 80 \times 30$ $\mathrm{mm}$; carbide four blade milling cutter model is HPL10.0 M, diameter is $10 \mathrm{~mm}$, the nominal spiral Angle $i=30^{\circ}$, radial rake angle $\gamma_{n}=0^{\circ}$, tool clearance angle $\alpha_{0}=20^{\circ}$, tooth wedge angle $\delta=70^{\circ}$. Experimental system consist of thedynamometer, multi-channel signal analyzer and computer. The 
model of multi-channel signal analyzer used in the test is DH5922N. The application of MFC AppWizard is based on dialog box data acquisition interface, which has a sampling frequency of 1000 Hz. Cutting speed $v_{\mathrm{c}}$ is $100 \mathrm{~m} / \mathrm{min}$, the spindle speed is $3200 \mathrm{r} / \mathrm{min}$, the axial cutting depth $a_{\mathrm{p}}$ is $0.5 \mathrm{~mm}$, each tooth feeding $f_{\mathrm{t}}$ is $0.025 \mathrm{~mm}$, the radial cutting width $a_{\mathrm{e}}$ is $10 \mathrm{~mm}$; Dry down slot milling is taken.

Experimental Result. Signal figure collected is shown in Figure 4. It can be seen from the graph that the maximum of $F_{\mathrm{x}}$ is around $160 \mathrm{~N}$; Cutting force fluctuation change, after removal not ideal signal curve, the minimum basic cutting force appears near the $0 \mathrm{~N}$, accords with the basic change law of milling forces.

\section{Thermalmechanical Analysis}

Method of Thermomechanical Analysis. The 3-D calculating model of cutting force in milling is established through the micro discrete method. The instantaneous chip thickness of micro element can be obtained by the geometric relations.

$h_{i}=f_{t} \sin \varphi_{k i} \Gamma_{k i}$

Where, $\varphi_{\mathrm{ki}}$ the case of infinitesimal rotation angle $i$ as shown in expression (14).

$\Gamma_{\mathrm{ki}} \quad$ judgment coefficient, as shown in expression (15).

$\varphi_{k i}=\theta(t)-\frac{y_{i}}{R_{i}} \tan \eta_{s}+(k-1) \frac{2 \pi}{N}$

Where, $k$ - the $\mathrm{k}_{\text {th }}$ blade.

$N$ the number of blades.

$R_{\mathrm{i}} \longrightarrow$ rotation radius of the $i_{\text {th }}$ element.

$\Gamma_{k i}=1$ (when the $i_{\text {th }}$ element of $k_{\text {th }}$ blade contacts workpiece)

Tangential, radial and axial cutting force of micro element of cutting blade can be respectively expressed by formulations $(16-18)^{[12]}$.

$d F_{t, \varphi_{k i}}=\left(K_{t c} h_{i}+K_{t e}\right) d y_{i}$

$d F_{r, \varphi_{k i}}=\left(K_{r c} h_{i}+K_{r e}\right) d y_{i}$

$d F_{a, \varphi_{k i}}=\left(K_{a c} h_{i}+K_{a e}\right) d y_{i}$

Where, $K_{t c} 、 K_{r c} 、 K_{a c}$ cutting force coefficient of tangential, radial and instantaneous axial respectively, can obtained by expressions $8 \sim 10$ considering $\Phi_{n}$ and material flow stress variation.

$K_{t e} 、 K_{r e} 、 K_{a e}$ tangential, radial and axial force coefficient on the edge, when the $h_{\mathrm{i}}$ equals zero, obtained by reverse cutting force test curve.

Thermomechanicalcalculation steps of vertical milling processing are shown as follows.

(1) determine the point of integral step length $\Delta \theta$, integration step $\Delta a_{p}$ of the blade in axial, and the cutting out angle $\Phi_{e x}\left(\Phi_{s t}=\operatorname{acos}\left(a_{e} / r-1\right), \Phi_{e x}=\pi\right)$.

(2) when the milling cutter rotation to arbitrary Angle $\theta(\mathrm{t})$, calculate the cutting forcedistribution.

(3) composite $\theta(\mathrm{t}), \Phi_{s t}$ and $\Phi_{e n}$ geometric relations, it can ensure the angle $\theta(\mathrm{t})$ in the $\mathrm{k}_{\mathrm{th}}$ blade, then calculate the cutting force for integral processing in their respective axial cutting depth. After this step, it can obtain the total cutting force of the $\mathrm{k}_{\mathrm{th}}$ blade.

(4) change $\theta(\mathrm{t})$, repeat step 2 and 3, it can get the cutting force changes when the blades rotating.

Analysis Results. For the study of thermalmechanical changes in end milling process, above thermomechanical calculation method is used. The same conditions and scheme as the experimental study is accepted. During Calculation, $\Delta \theta=5^{\circ}, \Delta a_{p}=0.025 \mathrm{~mm}$, the number of discrete points between shear line and the tool-chip contact line is 10 .

As the Fig.5shown, the change curve of cutting force with blade rotation within 1circle is obtained. From theresults of theFig. $5 \mathrm{a} \sim 5 \mathrm{c}$ can be seen that the cutting force $F_{\mathrm{x}}, F_{\mathrm{y}}$ and $F_{\mathrm{z}}$ in the main cutting plane show obvious periodic $(\pi / 2)$ variation. With the comparing of experimental and theoretical results, it hasa little phase lag (because of the response lag in the force transducer). The maximum $F_{x}$ of theoretical analysis is about $198 \mathrm{~N}$, the maximum $F_{y}$ is about $40 \mathrm{~N}$, the maximum $F_{z}$ is about $90 \mathrm{~N}$, which 
all appeared when the rotation angle equal to $\pi / 4$. The error between calculated and measured value of maximum $F_{x}, F_{y}$ and $F_{z}$ are $2 \%, 20 \%$ and $10 \%$ respectively, but the trend was consistent.

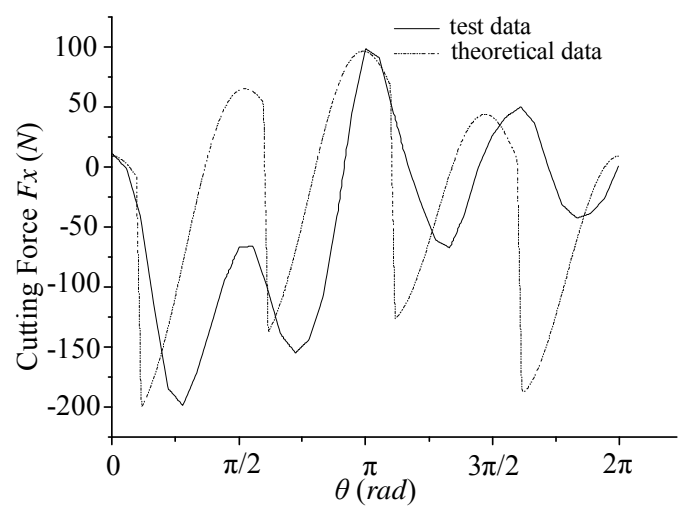

(a) Cutting Force $F_{x}$

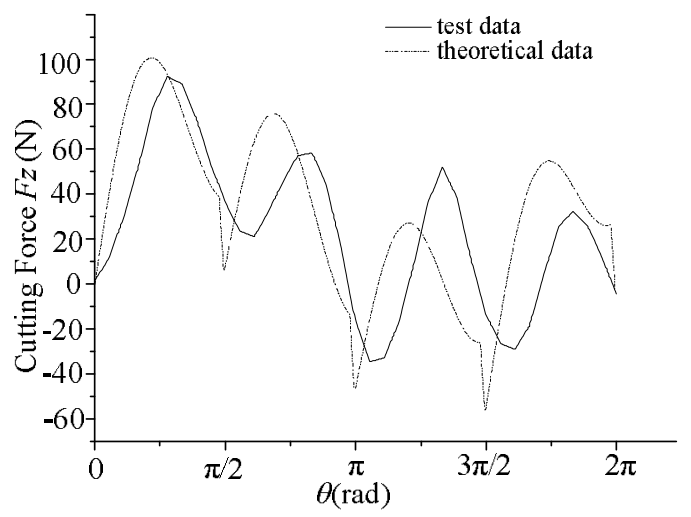

(c) Cutting Force $F_{z}$

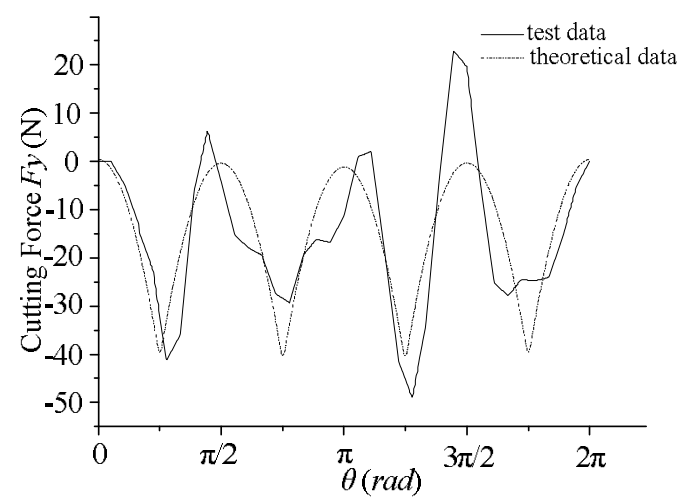

(b) Cutting Force $F_{y}$

Fig.5: Comparing of Results

\section{Summary}

(1) The steady-state oblique cutting thermal model is established; based on the above, the dynamical solving method of cutting force in the helical gear vertical milling processing was developed, the cutting force changes of AISI1045 steelunder specific conditions was studied, and the feasibility of the method is validated through the milling test.

(2) The proposed method not only takes the thermal effect of material flow stress change into account, but also the chip thickness influence on cutting force and cutting temperature.

(3) For whole cutting process, the maximum cutting force $F_{x}, F_{y}$ and $F_{z}$ are $198 \mathrm{~N}, 40 \mathrm{~N}$ and $90 \mathrm{~N}$ respectively, the error between the test data are $2 \%, 20 \%$ and $10 \%$ respectively, basically meet the requirements of thermal error of problem solving.

\section{Acknowledgments}

This work was financially supported by the Natural Science Foundation of Jiangsu Province of China (NO: BK20131341 and BK20150614).

\section{References}

[1] J. van der Geer, J.A.J. Hanraads, R.A. Lupton, The art of writing a scientific article, J. Sci. Commun. 163 (2000) 51-59.

Reference to a book:

[2] W. Strunk Jr., E.B. White, The Elements of Style, third ed., Macmillan, New York, 1979. 
Reference to a chapter in an edited book:

[3] G.R. Mettam, L.B. Adams, How to prepare an electronic version of your article, in: B.S. Jones, R.Z. Smith (Eds.), Introduction to the Electronic Age, E-Publishing Inc., New York, 1999, pp. 281-304.

[4] R.J. Ong, J.T. Dawley and P.G. Clem: submitted to Journal of Materials Research (2003)

[5] P.G. Clem, M. Rodriguez, J.A. Voigt and C.S. Ashley, U.S. Patent 6,231,666. (2001)

[6] Information on http://www.weld.labs.gov.cn

[1] SUHAIL A. H, ISMAIL N., WONG S.V., et al. Workpiece surface temperature for in-process surface roughness prediction using response surface methodology. Journal of Applied Sicences, 11(2011)308-315.

[2] OXLEY P.L.B.. Mechanics of Machining. Ellis Horwood press, Chichester, UK. (1989)

[3] MOUFKI A., DUDZINSKI D., MOLINARI A., et al. Thermo-viscoplastic modeling of oblique cutting : forces and chip flow predictions. International Journal of Mechanics Science, 42(2000)1205-1232.

[4] WU W.G.. Study on high efficiency precision cutting and its vibrating characteristic. Jiang Su University.(2007). (in Chinese)

[5] LI B.L., WANG X.L., HU J., et al. Thermomechanical modeling and simulation analysis of oblique cutting. China Mechanical Engineering, 21(2010)2401-2408.(inChinese)

[6] MOUFKI A., DEVILLEZ A., DUDZINSKI D., et al. Thermomechanical modelling of oblique cutting and experimental validation. International Journal of Machine Tools \& Manufacture, 44(2004)971-989.

[7] BURYTA D., SOWERBY R., YELLOWLEY I.. Stress distributions on the rake face during orthogonal machining. International Journal of Machine Tools \& Manufacture, 34(1994)721-739.

[8] DUDZINSKI D., MOLINARI A.. A modeling of cutting for viscoplasticmaterials. International Journal of Mechanics Science, 39(1997)369-389.

[9] MILTON C.S.. Metal Cutting Principles. Oxford University Press, New York, USA. (1984)

[10] MAUREL-PANTEL A., FONTAINE M., MICHEL G. et al. Experimental investigations from conventional to high speed milling on a 304-L stainless steel. International Journal of advanced manufacturing technology, 69(2013)2191-2213.

[11] FENG Y., WANG M.L., WANG B.S. et al. Research on Cutting Temperature of Workpiece in Milling Process Based on WPSO. Int J Adv Manuf Technol, 79(2015)427-435. 